\title{
Relaxed Graceful Labellings of Trees
}

\author{
Frank Van Bussel \\ Department of Computer Science \\ University of Toronto, Toronto, Ontario \\ fvb@cs.utoronto.ca
}

Submitted: June 22, 2001; Accepted: January 4, 2002

MR Subject Classification: 05C78

\begin{abstract}
A graph $G$ on $m$ edges is considered graceful if there is a labelling $f$ of the vertices of $G$ with distinct integers in the set $\{0,1, \ldots, m\}$ such that the induced edge labelling $g$ defined by $g(u v)=|f(u)-f(v)|$ is a bijection to $\{1, \ldots, m\}$. We here consider some relaxations of these conditions as applied to tree labellings:

1. Edge-relaxed graceful labellings, in which repeated edge labels are allowed,

2. Range-relaxed graceful labellings, in which the upper bound $m^{\prime}$ is allowed to go higher than the number of edges, and

3. Vertex-relaxed graceful labellings, in which repeated vertex labels are allowed.

The first of these had been looked at by Rosa and Širán̆ [10]. Here some linear bounds in the relevant metrics are given for range-relaxed and vertex-relaxed graceful labellings.
\end{abstract}

\section{Introduction}

Let $G$ be a graph with vertex set $V$ and edge set $E$, with $m=|E|$. The mapping $f(V) \rightarrow \mathbb{N}$ with the induced mapping $g(E) \rightarrow \mathbb{N}$ defined by $g(u v)=|f(u)-f(v)|$ is graceful if

(1) $f(V)$ is an injection to $\{0,1, \ldots, m\}$,

(2) $g(E)$ is a bijection to $\{1,2, \ldots, m\}$.

In the mid-1960's it was conjectured by Rosa [9] that all trees are graceful. This now notorious open problem has been know variously as Rosa's conjecture, Ringel's conjecture, or the graceful tree conjecture (GTC). While much work on graceful and related labellings has been done since then, progress on the GTC itself has been spotty at best; Anton Kotzig [7] has called it a "disease" of graph theory. 
Relaxations of graceful labelling have been investigated for as long as graceful labelling itself; Rosa himself presented several variants in [9]. Most of the work done, however, has been confined to graphs that are not graceful to begin with [2] [6]. It is only recently that relaxed graceful labellings of trees have come under scrutiny, with the results of Rosa and Širáñ on the gracesize of trees [10], and Ringel, Llado, and Serra's study of bigraceful trees [8].

For terms and definitions from general graph theory the reader is referred to West [12].

\section{A schematization of relaxed graceful labellings}

While there are innumerable relaxations of gracefulness we can choose from, in this paper we will restrict ourselves to three that could be considered the most basic, in that the properties of a graceful labelling stated above are weakened without affecting the distinctive relationship between the vertex and edge labellings:

1. Edge-relaxed: Property (2) is relaxed, in that $g$ need not be a bijection but can be any mapping from $E$ to $\{1, \ldots, m\}$.

2. Range-relaxed: Properties (1) and (2) above are relaxed, in that the upper bound on $f$ and $g$ is allowed to be some $m^{\prime} \geq m$. The mapping $g$ need no longer be a bijection, but must still be an injection.

3. Vertex-relaxed: Property (1) is relaxed, allowing $f$ to be any mapping from $V$ to $\{0, \ldots, m\}$.

Edge-relaxed graceful labelling is in a sense the most simple and natural, in that any proper labelling of the vertices of a graph $G$ on $m$ edges with numbers in the range $\{0, \ldots, m\}$ satisfies the requirements. It is these labellings that were studied by Rosa and Siráň in their 1995 paper [10]. They were able to show that all trees on $m$ edges have an edge-relaxed graceful labelling with at least $\frac{5}{7} m$ of the edge labels distinct.

Range-relaxed graceful (RRG) labelling, our second scheme, has a much more extensive history; it has been brought forth and fiddled with under various guises since the graceful labelling problem was first popularized by Solomon Golomb in the early 1970's. Since that time we have seen $k$-graceful, node-graceful, nearly graceful, almost graceful and pseudograceful labellings (see [5]), which are all comprehended in one way or another under the range-relaxed scheme. Since not all labellings in even the most extended range need be range-relaxed graceful, one could say that labellings of this sort are "not as relaxed" as their edge-relaxed counterparts. However, as is the case with edge-relaxed graceful labellings, every graph has a range-relaxed graceful labelling in some range; if, for example, the vertices are labelled with distinct powers of 2 , the induced edge labels must all be distinct. This of course is definitely not the best we can do-the cycle $C_{n}$ is not graceful when $n$ is equivalent to 1 or $2(\bmod 4)$, but it always has a RRG labelling in the range $\{0, \ldots, n+1\}$. 
Vertex-relaxed graceful (VRG) labellings were apparently studied in the 1970's by Bermond and Lehel in connection with graceful labellings of windmill graphs [1], but since then there does not seem to have been any work done with them. The closest recent antecedent is the bigraceful labelling scheme for bipartite graphs, from Ringel, Llado, and Serra [8]; these labellings satisfy property (2) but only require that vertex labels be unique within each bipartition set. Other variations of gracefulness which relax distinctness conditions (such as equitable labellings) introduce new constraints as well.

While in a sense vertex-relaxed graceful labellings can be thought of as "dual" to edge-relaxed graceful labellings, in structure a VRG labelling tends to look much more like a proper graceful labelling; for example, vertices labelled 0 and $m$ must be present and adjacent, and $m-1$ adjacent to 0 or 1 adjacent to $m$, and so on. Many graphs that are too sparse to be graceful have a VRG labelling; any matching on $m$ edges, for example, can be so labelled by giving 0 to one endpoint of each edge, and assigning $1, \ldots, m$ arbitrarily to the other endpoints. Not all graphs have a VRG labelling, however, and in fact we have yet to encounter a connected ungraceful graph which is vertex-relaxed graceful.

In this paper the following two theorems will be presented regarding the relaxed graceful labelling of trees:

Theorem 1 Every tree $T$ on $m$ edges has a range-relaxed graceful labelling $f$ with vertex labels in the range $0, \ldots, 2 m$-diameter $(T)$.

Theorem 2 Every tree on $n$ vertices has a vertex-relaxed graceful labelling such that the number of distinct vertex labels is strictly greater than $\frac{n}{2}$.

\section{Proof of Theorem 1}

Let $v_{0}$ be an arbitrary vertex of $T$, and consider $T$ in its usual representation as a tree rooted at $v_{0}$; that is, vertices the same distance from $v_{0}$ are drawn on the same level, and edges are not allowed to cross each other. We will assume that the longest path from $v_{0}$ is leftmost in this representation. Let the number of vertices in this longest path be $l$, let the vertex on this path at level $i$ be denoted as $v_{i}, i=0,1, \ldots, l-1$, and let the number of vertices on the $i$-th level be denoted as $k_{i}$. The following construction provides a labelling $f$ of $V(T)$ in the range $\{0, \ldots, 2 m-l+1\}$.

1. The root vertex $v_{0}$ takes a provisional value $\alpha$; once the range of values has been established we will shift all labels by a constant such that the lowest label is 0 . We give the label $\alpha+1$ to $v_{1}$, the leftmost child of $v_{0}$.

2. For $i>1$ each vertex $v_{i}$ on the leftmost path receives the labelling

$$
f\left(v_{i}\right)= \begin{cases}f\left(v_{i-2}\right)-k_{i-2}-k_{i-1}+1=\alpha-\sum_{j=0}^{i-1} k_{j}+\frac{i}{2} & \text { if } i \text { is even } \\ f\left(v_{i-2}\right)+k_{i-2}+k_{i-1}-1=\alpha+\sum_{j=0}^{i-1} k_{j}-\frac{i-1}{2} & \text { if } i \text { is odd }\end{cases}
$$


3. A vertex $u$ on the $i$-th level $k$ places to the right of $v_{i}, 0 \leq k \leq k_{i}-1$, receives the labelling

$$
f(u)= \begin{cases}f\left(v_{i}\right)-k & \text { if } i \text { is even } \\ f\left(v_{i}\right)+k & \text { if } i \text { is odd }\end{cases}
$$

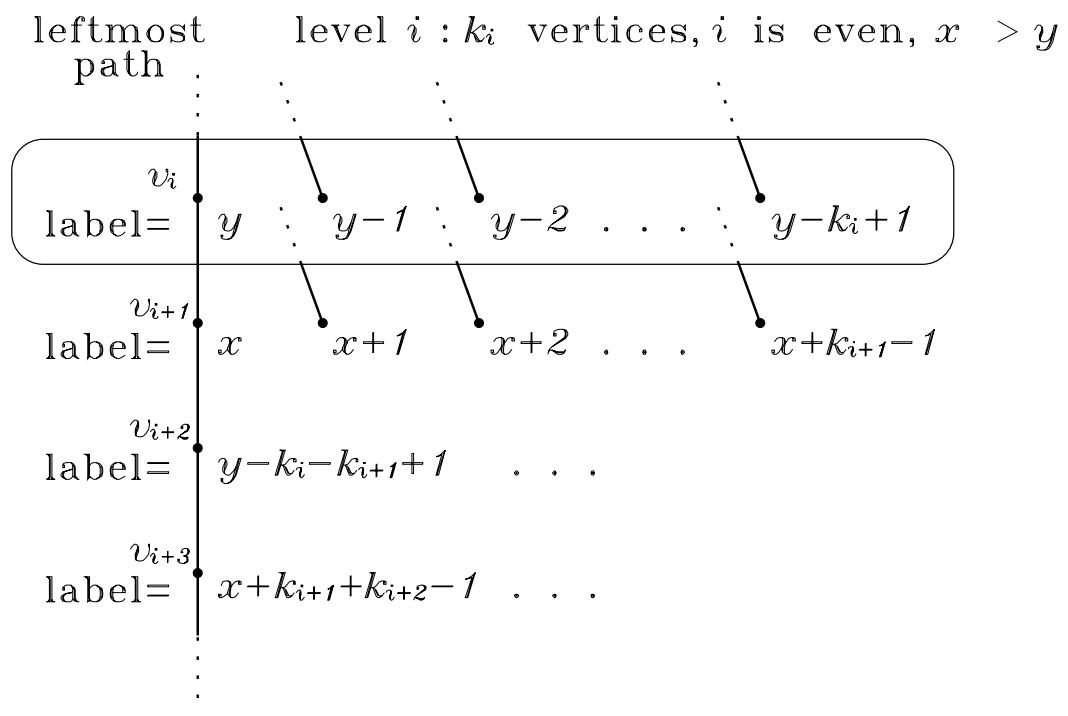

Figure 1: Construction for Theorem 1

By the construction all vertex labels are distinct, since on even levels they are monotonically decreasing as we go from left to right and from top to bottom, while on the odd levels they are increasing. Figure 1 shows how these two labelling schemes are interlaced. Likewise, the edge labelling $g$ of $E(T)$ is monotonically increasing going from left to right and from top to bottom in the tree:

1. Consider two edges $u_{i} u_{i+1}$ and $w_{i} w_{i+1}$ between vertices on consecutive levels $i$ and $i+1$, where $u_{i} u_{i+1}$ is left of $w_{i} w_{i+1}$ and $i$ is even. By part (3) of the construction, since edges cannot cross we have $f\left(u_{i}\right)>f\left(w_{i}\right)$ and $f\left(u_{i+1}\right)<f\left(w_{i+1}\right)$; hence

$$
g\left(u_{i} u_{i+1}\right)=f\left(u_{i+1}\right)-f\left(u_{i}\right)<f\left(w_{i+1}\right)-f\left(w_{i}\right)=g\left(w_{i} w_{i+1}\right) .
$$

The same follows for $i$ odd by an analogous proof.

2. Consider any two edges $u_{i} u_{i+1}$ between levels $i$ and $i+1$ and $w_{i+1} w_{i+2}$ between levels $i+1$ and $i+2$ where $i$ is again even. Since the rightmost vertices $r_{i}$ on level $i$ and $r_{i+1}$ on level $i+1$ are respectively the minimum and maximum label on levels $i$ and $i+1$, we have that

$$
f\left(r_{i+1}\right)-f\left(r_{i}\right) \geq g\left(u_{i} u_{i+1}\right)
$$


while the leftmost difference $g\left(v_{i+1} v_{i+2}\right)$ is a lower bound on the labels of the edges between levels $i+1$ and $i+2$. Hence we have

$$
\begin{aligned}
& g\left(u_{i} u_{i+1}\right) \leq f\left(r_{i+1}\right)-f\left(r_{i}\right)=f\left(v_{i+1}\right)+k_{i+1}-1-\left(f\left(v_{i}\right)-k_{i}+1\right) \\
& <f\left(v_{i+1}\right)-\left(f\left(v_{i}\right)-k_{i}-k_{i+1}+1\right)=g\left(v_{i+1} v_{i+2}\right) \\
& \leq g\left(w_{i+1} w_{i+2}\right) \text {. }
\end{aligned}
$$

Again, this holds for $i$ odd with minor modifications.

Lastly, we check that the bound on the range holds. Let $f_{M I N}$ and $f_{M A X}$ be respectively the minimum and maximum vertex labels generated by the labelling $f$. In the case where $l$ is even, the highest labelled vertex is the rightmost on level $l-1$, and the lowest labelled vertex is the rightmost on level $l-2$; hence by the construction

$$
\begin{aligned}
f_{M A X} & =f\left(v_{l-1}\right)+k_{l-1}-1 \\
& =\alpha+\sum_{j=0}^{l-2} k_{j}-\frac{l-2}{2}+k_{l-1}-1 \\
& =\alpha+m+1-\frac{l}{2} . \\
f_{M I N} & =f\left(v_{l-2}\right)-k_{l-2}+1 \\
& =\alpha-\sum_{j=0}^{l-3} k_{j}+\frac{l-2}{2}-k_{l-2}+1 \\
& =\alpha-m-1+k_{l-1}+\frac{l}{2} .
\end{aligned}
$$

From this we obtain the bound on the range:

$$
\begin{aligned}
f_{M A X}-f_{M I N} & =\alpha+m+1-\frac{l}{2}-\left(\alpha-m-1+k_{l-1}+\frac{l}{2}\right) \\
& =2 m-l+2-k_{l-1} \\
& \leq 2 m-l+1 .
\end{aligned}
$$

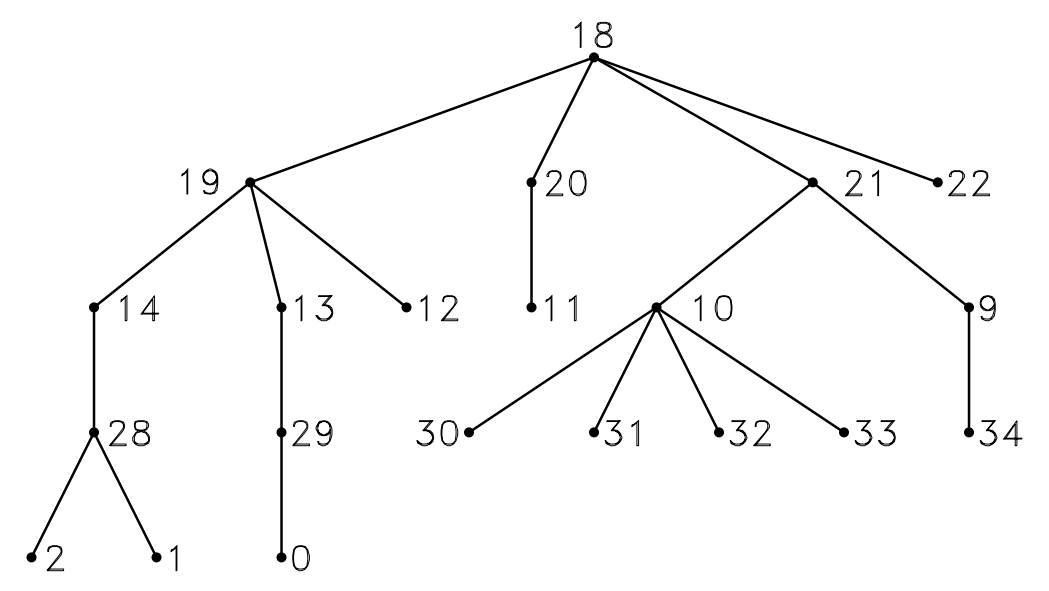

Figure 2: RRG labelling of a tree using Theorem 1 construction

By choosing for our root vertex one endpoint of a longest path in $T$, we can obtain a labelling in the range $2 m-$ diameter $(T)$. 
We note that this bound is tight for paths, and is "good" for long, stringy trees; but for trees of low fixed diameter we still have not broken $(2-\varepsilon) m$. There do exist variants of the above construction that in practice seem to always give us a range well within $\frac{3}{2} m$; these, however, assign labels opportunistically rather than monotonically, and have not as of yet given us any rigourous results.

\section{Proof of Theorem 2}

To establish Theorem 2 it is enough to show that every tree has a vertex-relaxed graceful labelling where all vertex labels in the larger of the bipartition sets are distinct; for this

we will need a little bit of new machinery. An often studied subset of graceful labellings are bipartite graceful (or $\alpha$-graceful) labellings; the labelling $f$ of $G$ is bipartite if the vertices of $G$ have a bipartition into sets $A$ and $B$ such that there exists a constant $\alpha \in \mathbb{N}$ satisfying, for every vertex $v \in A$

$$
f(v) \leq \alpha,
$$

and for every vertex $v \in B$

$$
f(v)>\alpha .
$$

If $G$ is a tree then $\alpha$ is of course the highest label in the low partition $A$, and is equal to $|A|-1$. Among the many useful properties of this extra condition is that if $f$ is a bipartite graceful labelling of a graph $G$ then addition of a constant to every vertex label in the high bipartition set results in addition of the same constant to every edge label. We now consider a weakened version of bipartite labelling, in that the above condition is applied (with some modifications) not globally but locally; the highest label in the low partition as a consequence loses its defining status.

Definition 3 Let $G$ be a graph with a labelling $f$. We say that $f$ is locally bipartite if the vertices of $G$ have a bipartition into sets $A$ and $B$ such that for every vertex $v \in A$

$$
\forall u \in N(v), \quad f(v)<f(u)
$$

and for every vertex $v \in B$

$$
\forall u \in N(v), \quad f(v)>f(u)
$$

where $N(v)$ is the open neighbourhood of $v$.

Locally bipartite labellings were introduced independently in [4] and [11]. For our present purposes we are interested in the fact that like fully bipartite labellings they have the property that the value of all edge labels can be shifted up by a constant simply by adding that constant to the labels of all vertices in the high partition. 
Claim 4 Let $T$ be a tree, with the sets $A$ and $B$ a bipartition of the vertices of $T$, and $v$ an arbitrary vertex in $A$. There exists a vertex-relaxed graceful labelling $f$ of $T$ which satisfies the following properties as well:

1. The labelling $f$ is locally bipartite, with $B$ being the high partition;

2. The vertex $v$ is assigned the label 0 by $f$;

3. The labels of all vertices in $B$ are distinct.

Such a labelling will be denoted as $\mathrm{VRG}^{\prime}$ with respect to $v$.

Proof: Assume that all trees on less that $n$ vertices satisfy the claim, and let $T$ be any tree on $n$ vertices and $m$ edges. For every vertex $v$ in $V(T)$, we can construct a labelling $f$ that is $\mathrm{VRG}^{\prime}$ with respect to $v$ in the following manner:

CASE: The degree of $v$ is at least 2. Then $T$ can be split at $v$ to form two trees $T_{1}$ and $T_{2}$ of orders strictly less than $n$ and greater than 1 . Let $v_{1}$ and $v_{2}$ be the vertices of $T_{1}$ and $T_{2}$ respectively which are identified to form $v$ in $T$, and let $\left(A_{1}, B_{1}\right)$ and $\left(A_{2}, B_{2}\right)$ be bipartitions of $T_{1}$ and $T_{2}$ such that $v_{1} \in A_{1}$ and $v_{2} \in A_{2}$.

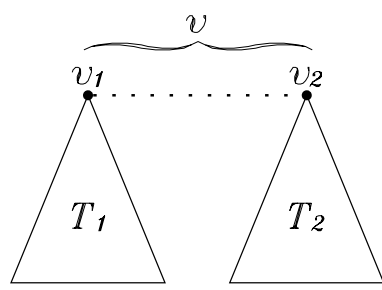

Figure 3: The degree of $v$ is at least 2

By our assumption $T_{1}$ and $T_{2}$ possess $\mathrm{VRG}^{\prime}$ labellings $f_{1}$ and $f_{2}$ where $v_{1}$ and $v_{2}$ are assigned the label 0 . If the number of edges in $T_{1}$ is $m_{1}$ the labelling $f$ of $T$ defined by

$$
f(u)= \begin{cases}f_{1}(u) & \text { if } u \in V\left(T_{1}\right), \\ f_{2}(u) & \text { if } u \in A_{2}, \\ f_{2}(u)+m_{1} & \text { if } u \in B_{2} .\end{cases}
$$

is $\mathrm{VRG}^{\prime}$ with respect to $v$. Since $f_{1}$ and $f_{2}$ are $\mathrm{VRG}^{\prime}$ with respect to $v_{1}$ and $v_{2}$ respectively, we have:

1. $f(v)=f_{1}\left(v_{1}\right)=f_{2}\left(v_{2}\right)=0$.

2. $f_{1}$ and $f_{2}$ are both locally bipartite; addition of a constant to the labels in the high partition does not change that fact for $f_{2}$, nor does amalgamation of the subtrees at $v$, so $f$ is as well locally bipartite. 
3. The edge labels generated by $f$ in $T_{1}$ are the same as those generated by $f_{1}$ : $\left\{1, \ldots, m_{1}\right\}$. The edge labels generated by $f$ in $T_{2}$ are those generated by $f_{2}$ shifted up by the constant $m_{1}:\left\{m_{1}+1, \ldots, m_{1}+\left|E\left(T_{2}\right)\right|\right\}$. Hence $f$ is a vertex relaxed graceful labelling of $T$.

4. All labels given by $f_{1}$ to $B_{1}$ are distinct, as are all labels given by $f_{2}$ to $B_{2}$. Since $f_{2}$ is locally bipartite no vertex in $B_{2}$ is given the label 0 by $f_{2}$. Hence

$$
\min \left\{f_{2}\left(B_{2}\right)\right\}+m_{1} \geq m_{1}+1>m_{1}=\max \left\{f_{1}\left(B_{1}\right)\right\} .
$$

Therefore all labels given to $B_{1} \cup B_{2}$ by $f$ are distinct.

CASE: The degree of $v$ is 1 . Let $w$ denote the sole neighbour of $v$. If $T$ is not $P_{2}$ (which definitely satisfies our claim), then $w$ has $k \geq 1$ other neighbours $r_{1}, r_{2}, \ldots, r_{k}$. Let the trees of $T-\{w\}$ rooted at each $r_{i}$ be denoted by $T_{i}$, their size by $m_{i}$, and bipartition by $\left(A_{i}, B_{i}\right)$ with $r_{i} \in A_{i}$.

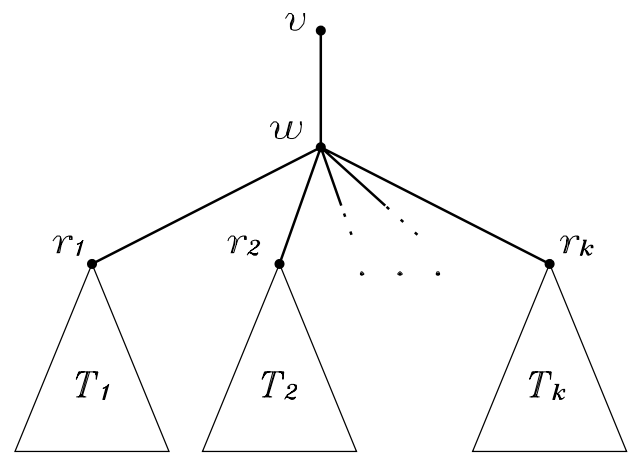

Figure 4: The degree of $v$ is 1

Since no $T_{i}$ is of order greater than $n-2$, by our assumption each has a $\mathrm{VRG}^{\prime}$ labelling with respect to $r_{i}$ which we will denote $f_{i}$. A labelling $f$ of $T$ which is $\mathrm{VRG}^{\prime}$ with respect to $v$ is then given by:

$$
\begin{array}{rlrl}
f(v) & =0, & \\
f(w) & =m, & & \\
f(u) & =f_{i}(u)+i & & \text { if } u \in A_{i}, \\
& =f_{i}(u)+\sum_{j=1}^{i-1} m_{j}+i & & \text { if } u \in B_{i} .
\end{array}
$$

We verify that this is $\mathrm{VRG}^{\prime}$; in the following $M_{i}$ will denote the sum $\sum_{j=1}^{i-1} m_{j}$ :

1. $f(v)$ is explicitly assigned 0 by the construction.

2. Each $f_{i}$ is locally bipartite; the effect of $f$ on $f_{i}$ is to add the constant $i$ to all labels of $V\left(T_{i}\right)$, and the additional constant $M_{i}$ to the labels of the high partition $B_{i}$, hence $f$ applied to each $T_{i}$ is locally bipartite. Since $f\left(r_{i}\right)$ is the lowest label in each $T_{i}$, and $f(w)$ of course the highest in $T, f$ is locally bipartite with respect to $T$ as well. 
3. Since the addition of a constant to all vertex labels does not effect the edge labels generated, we have that $f$ applied to each $T_{i}$ generates $\left\{M_{i}+1, \ldots, M_{i}+m_{i}\right\}$; since $M_{1}$ equals 0 and $M_{i+1}$ equals $M_{i}+m_{i}$, these labels with respect to the $T_{i}$ 's are distinct and cover $\left\{1, \ldots, M_{k}+m_{k}\right\}$. Since $f\left(r_{i}\right)$ equals $i$ the edges labels generated in the subgraph of $T$ induced by $\left\{v, w, r_{1}, \ldots, r_{k}\right\}$ are simply $\{m-k, m-k+1, \ldots, m\}$. The number of edges of $T$ not in this induced subgraph is $M_{k}+m_{k}$, hence $f$ is vertex-relaxed graceful.

4. For each $f_{i}$ we have all vertex labels in $B_{i}$ distinct, with $\max \left\{f_{i}\left(B_{i}\right)\right\}$ at most $m_{i}$ and $\min \left\{f_{i}\left(B_{i}\right)\right\}$ at least 1 . Hence we have that

$$
\max \left\{f\left(B_{i}\right)\right\} \leq M_{i}+m_{i}+i<M_{i+1}+i+1 \leq \min \left\{f\left(B_{i+1}\right)\right\} .
$$

Therefore the labels $f$ assigns to $\bigcup_{i=1}^{k} B_{i}$ are all distinct. The maximum of these is at most $M_{k}+m_{k}+k=m-1$, so all labels in the high partition of $T$ are distinct and $f$ is $\mathrm{VRG}^{\prime}$ with respect to $v$.

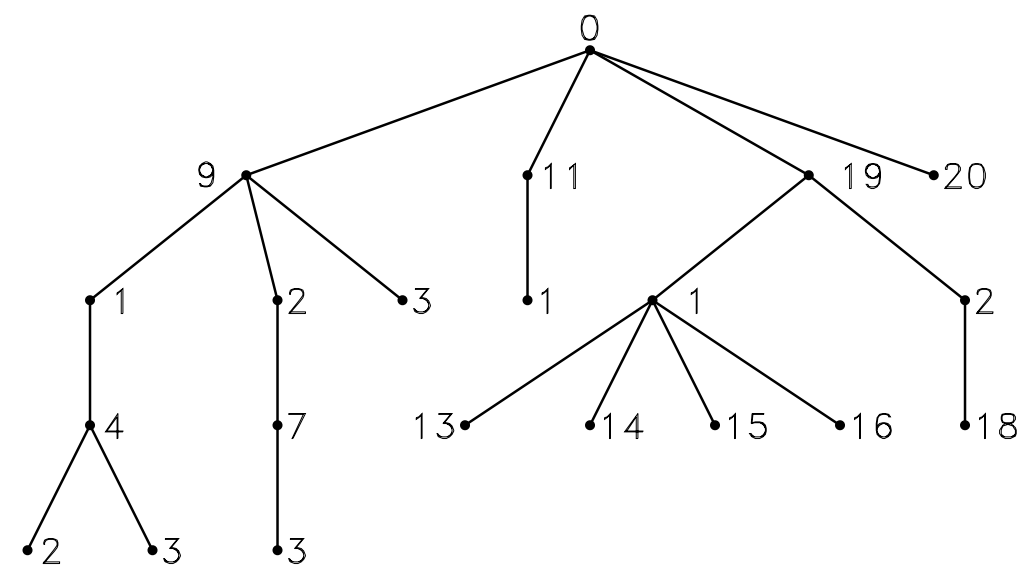

Figure 5: VRG labelling of a tree using construction from Claim 4

Of course, to obtain the bound stated in Theorem 2, we choose a vertex $v$ in $V(T)$ such that the set $A$ is the smaller one of the bipartition. We can then obtain a labelling $f$ that is $\mathrm{VRG}^{\prime}$ with respect to $v$ by applying the above construction recursively. As mentioned before, the label 0 cannot be used for any vertex in $B$; hence we have

$$
|f(V(T))| \geq|B|+1 \geq \frac{n}{2}+1 .
$$




\section{$5 \quad$ Closing remarks}

\subsection{Better bounds}

Even if the graceful tree conjecture remains unattainable, better bounds for range-relaxed and vertex-relaxed graceful labellings of trees should be within reach. The techniques employed here, however, may not take us much further, since our results depend in part upon the tree's diameter for RRG labellings and the difference in the size of its bipartition sets for VRG labellings; both may be arbitrarily small in relation to the size of the tree. Hence different approaches will probably need to be taken in order to obtain bounds that are, for example, comparable to those of Rosa and Širán for edge-relaxed graceful labellings.

Question: For a tree $T$ on $m$ edges and $n=m+1$ vertices, is there any $\varepsilon>0$ for which we can guarantee a range-relaxed graceful labelling within the range $(2-\varepsilon) m$, or a vertex-relaxed graceful labelling with at least $\left(\frac{1}{2}+\varepsilon\right) n$ distinct vertex labels? In particular, can we obtain bounds of roughly $\frac{7}{5} m$ and $\frac{5}{7} n$ respectively?

\subsection{Possible reductions}

A related question is whether there are reductions from any one of these labellings to any other which preserves to some extent the bound achieved in each individual case. At present we do know that if a tree $T$ on $n$ vertices has a vertex-relaxed graceful labelling $f$ with $c n$ distinct vertex labels we can obtain a range-relaxed graceful labelling in the range $n-1$ for at least one tree $T^{\prime}$ of order $c n$. While it is a known fact that a subgraph of a graceful graph is not necessarily graceful, if a graph $G$ on $m$ edges is graceful every subgraph of $G$ has a range-relaxed graceful labelling in range $\{0, \ldots, m\}$; on the other hand, if we have a graph $H$ with a vertex-relaxed graceful labelling $f_{H}$ we can identify any two vertices with the same label to obtain a VRG labelled graph $H^{\prime}$, since duplicate edges and self-loops are never introduced by such vertex indentifications. Hence we can achieve our "weak" reduction by identifying all like-labelled vertices $f$ gives to $T$ and then extracting a spanning tree from the resulting graph.

Question: Do there exist direct reductions between edge-relaxed, range-relaxed, and vertex-relaxed graceful labellings for a given tree T? If so, would any such reduction help us to significantly tighten our best known bounds in terms of $|E(T)|$ and $|V(T)|$ ?

\subsection{Graceful versus vertex-relaxed graceful}

Lastly, the idea that for connected graphs vertex-relaxed gracefulness may be strongly correlated with gracefulness proper has very intriguing consequences. If a graph has too few edges to be graceful then it is not connected in the first place; as mentioned earlier, in this case there is often a VRG labelling. With connected graphs the situation is quite different. While a full investigation of this must wait, it is certainly worth looking briefly at how vertex-relaxation fares against some known classes of ungraceful connected graphs: 
1. A parity condition given by Rosa [9], that any Eulerian graph $G$ on $m$ edges has no graceful labelling if $m$ is equivalent to 1 or $2(\bmod 4)$, applies as much in the VRG case as when all vertices have distinct labels. This is because Eulerian graphs can be decomposed into cycles, and the sum of the edge labels on any cycle must be even.

2. With dense graphs, allowing duplicate vertex labels offers no advantage; for a graph to have any VRG labelling that is not simply graceful at least two vertices with disjoint closed neighbourhoods must be present (otherwise, we will have two vertices with the same label both adjacent to some third vertex, or adjacent to each other).

3. Similarly, the presence of any dominant vertices precludes the use of duplicate labels, hence French 2 and 3 windmills are not vertex-relaxed graceful despite not being Eulerian or too dense; when $k$ is greater than 3 the only vertex-relaxed graceful labellings of French $k$-windmills are proper graceful labellings.

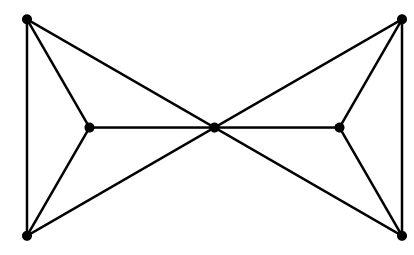

Figure 6: French 2-windmill

Question: Do all connected graphs that have a vertex-relaxed graceful labelling have a proper graceful labelling?

We note that if the above question can be answered in the affirmative then the graceful tree conjecture immediately follows.

\section{References}

[1] J-C. Bermond. Graceful graphs, radio antennae, and French windmills. In R.J. Wilson, editor, Graph Theory and Combinatorics, pages 18-37. Pitman Publishing Ltd., 1979.

[2] G.S. Bloom and S.W. Golomb. Numbered complete graphs, unusual rulers, and assorted applications. In Theory and Applications of Graphs, number 642 in Lecture Notes in Math., pages 53-65. Springer-Verlag, 1978.

[3] C.P. Bonnington and J. Širáň. Bipartite labelings of trees with maximum degree three. Journal of Graph Theory, 31:7-15, 1999. 
[4] S. El-Zanati, M. Kenig, and C. Vanden Eynden. Near $\alpha$-labelings of bipartite graphs. Australasian Journal of Combinatorics, 21:275-285, 2000.

[5] J.A. Gallian. A dynamic survey of graph labeling. Electronic Journal of Combinatorics. Dynamic Surveys, DS6, 95 pages (December 6, 2001).

[6] S.W. Golomb. How to number a graph. In R.C. Read, editor, Graph Theory and Computing, pages 23-37. Academic Press, 1972.

[7] C. Huang, A. Kotzig, and A. Rosa. Further results on tree labellings. Utilitas Mathematica, 21C:31-48, 1982.

[8] G. Ringel, A. Llada, and O. Serra. Another tree conjecture. Bulletin of the Institute of Combinatorics and its Applications, 18:83-85, 1996.

[9] A. Rosa. On certain valuations of the vertices of a graph. In Theory of Graphs, International Symposium, Rome, July 1966, pages 349-355. Gordon and Breach, NY, 1967.

[10] A. Rosa and J. Širáň. Bipartite labelings of trees and the gracesize. Journal of Graph Theory, 19:201-215, 1995.

[11] F. Van Bussel. Towards the graceful tree conjecture. MSc thesis, University of Toronto, Computer Science Dept., 2000.

[12] D. West. Introduction to Graph Theory. Prentice Hall, 1996. 